\title{
Re-Analysis of 165 Amplicon Sequencing Data Reveals Soil Microbial Population Shifts in Rice Fields under Drought Condition
}

Seok-Won Jang ${ }^{1,2+}$, Myeong-Hyun Yoou ${ }^{3+}$, Woo-Jong Hong ${ }^{3+}$, Yeon-Ju Kim ${ }^{4}$, Eun-Jin Lee ${ }^{1^{*}}$ and Ki-Hong Jung ${ }^{3^{*}}$ (D)

\begin{abstract}
Rice (Oryza sativa. L) has been intensively studied to ensure a stable global supply of this commodity in the face of rapid global climate change. A critical factor that decreases crop yield is drought, which has been analyzed in various ways through many researches. Microbiome-based studies of rice investigate the symbiosis between rice and bacteria, which has been proposed as a way to overcome problems caused by drought. Several rice-associated metagenomic profiles obtained under drought conditions have been reported since the advent of next generation sequencing (NGS) technology. To elucidate the future diversity of plants and microorganisms and to promote sustainable agriculture, we reanalyzed 64 of the publicly available $16 \mathrm{~S}$ amplicon sequencing data produced under drought condition. In the process of integrating data sets, however, we found an inconsistency that serves as a bottleneck for microbiome-based sustainability research. While this report provides clues about the composition of the microbiome under the drought conditions, the results are affected by differences in the location of the experiments, sampling conditions, and analysis protocols. Re-analysis of amplicon sequencing data of the soil microbiome in rice fields suggests that microbial composition shifts in response to drought condition and the presence of plants. Among the bacteria involved, the phylum Proteobacteria appears to play the most important role in the survival of rice under drought condition.
\end{abstract}

Keywords: Meta-analysis, Rice, Drought, Microbiome, Amplicon sequencing

\section{Findings}

There is much research aiming to improve rice yields in order to feed a rapidly growing human population and improve its tolerance to climate change. In particular, drought stress, which is associated with global warming and desertification, greatly reduces rice production (Peng et al. 2004). Considerable progress has been made in identifying traits

\footnotetext{
* Correspondence: eunjinlee@korea.ac.kr; khjung2010@khu.ac.kr

'Seok-Won Jang, Myeong-Hyun Yoou and Woo-Jong Hong contributed equally to this work.

${ }^{1}$ Division of Life Sciences, College of Life Sciences and Biotechnology, Korea University, Seoul 02841, South Korea

${ }^{3}$ Graduate School of Biotechnology \& Crop Biotech Institute, Kyung Hee University, Yongin 17104, South Korea

Full list of author information is available at the end of the article
}

that promote drought tolerance in rice. However, it has not yet been possible to actually apply this knowledge due to the tradeoff between drought resistance and yield.

Advances in next generation sequencing (NGS) technology has made it possible to rapidly collect large amounts of genetic information. Among the various sequencing techniques, the development of amplicon sequencing of the 16S rRNA gene in prokaryotes has led to the field of metagenome analysis. Even before the advent of NGS sequencing techniques, interactions between plant and root microorganisms have been studied (Lethbridge and Davidson 1983; Germida et al. 1998; Brencic and Winans 2005). However, the use of 
amplicon sequencing methods has accelerated analysis of this interaction.

Diverse microbes interact with plants (Lugtenberg and Kamilova 2009; Berg et al. 2014). Thus, the power of amplicon sequencing analysis in rice has been applied to better understand the relationship between plants and microbes in the underground areas, namely, the soil, rhizosphere, rhizoplane, and endosphere (Arjun and Harikrishnan 2011; Sessitsch et al. 2012; Edwards et al. 2015). Moreover, microbiome analyses have proven useful in understanding the effects of external factors such as abiotic stress and fertilizer limitation (Ahn et al. 2012; Ikeda et al. 2014; Rothenberg et al., 2016). Amplicon sequencing has also been applied to rice under drought conditions (Santos-Medellin et al. 2017; Reim et al. 2017). However, amplicon sequencing data from different studies vary due to geographical differences and the presence or absence of plant. To better understand the relationship between the microbiome and drought condition, which are closely linked to rice productivity, we reanalyzed publicly available amplicon sequencing data on the soil microbiome of rice under drought condition. We identify a candidate phylum that plays a role in the plant's response to the limits imposed by drought conditions on rice growth.

We collected 2061 microbiome sequence datasets from different plant species, sorted the data according to the workflow, and reanalyzed 64 soil microbiome sequences of rice (Fig. 1, Table S1). The microbiome data consists of data from soil, endosphere, rhizosphere, and rhizoplane (Table S1); however, the amount of microbiome data from the endosphere, rhizosphere, and rhizoplane is insufficient to allow rigorous comparison and re-analysis. Thus, we utilized only soil microbiome data for our analysis. We compared four different normalization methods: minmax, median, average, and total count, all of which showed similar patterns (Tables S2, S3, S4, and S5). Using normalized data from each sample, we selected the top 16 most abundant phyla (Table 1).

Analysis of a stacked bar plot and alpha diversity boxplot (Fig. 2, and Figure S1) indicates that the predominant members of the microbiome under drought conditions changed according to the presence or absence of plants. A two-way ANOVA test confirms the significance of this microbiome shift in response to plants (Table S6). In addition, we compared the relative abundance levels between members of the microbiome community in different environmental conditions (Table S7). Based on the shift pattern of phyla, this analysis yielded the following four clusters: phyla affected by both factors, those not affected by both factors, those affected by only the presence of plants, and those affected by only drought condition (Table S6). Among these clusters, Cyanobacteria and Epsilonbacteraeota were not affected by either factor. Meanwhile, Acidobacteria, Actinobacteria, Euryarchaeota,

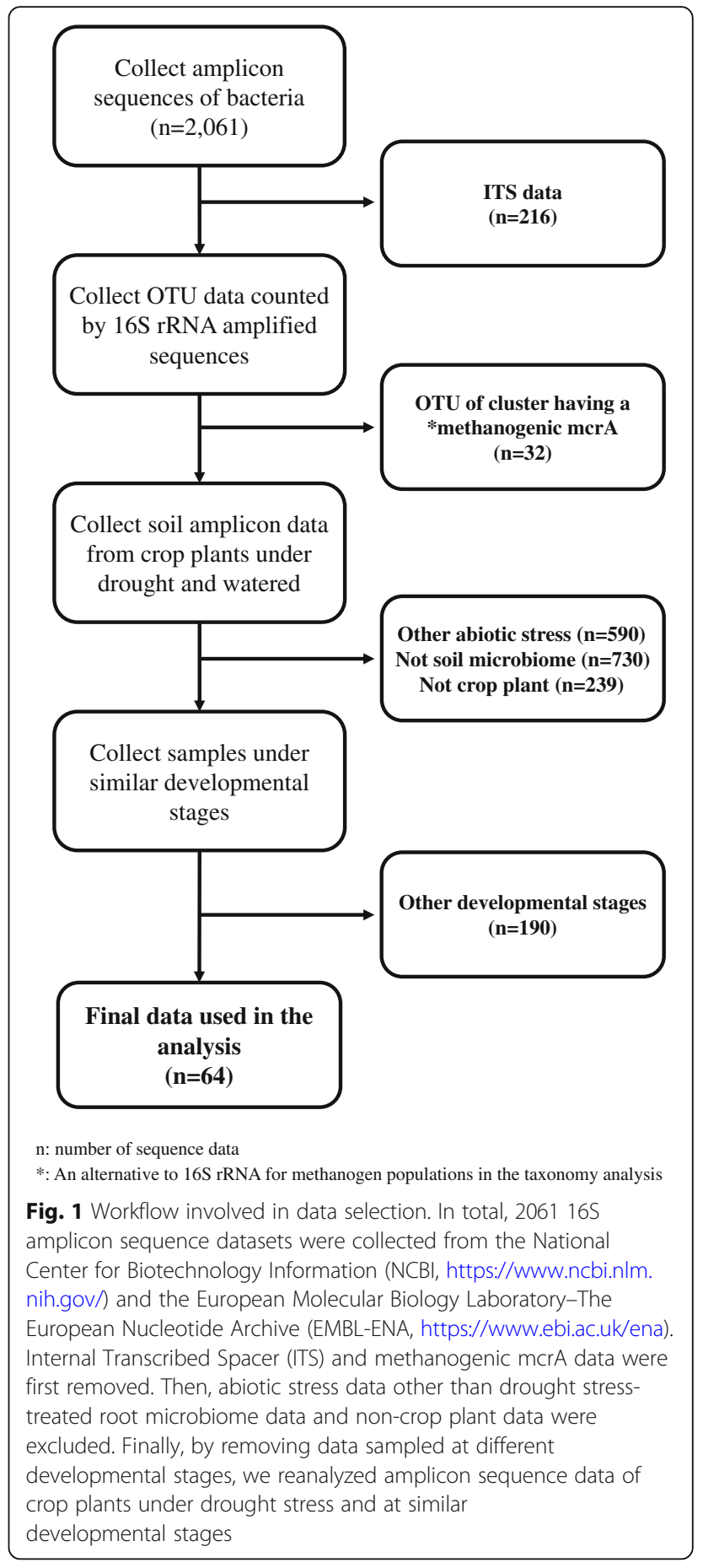

Gemmatimonadetes, and Planctomycetes were affected by only one factor, i.e., the presence of plants. To focus only on soil microbiomes affected by drought conditions, we analyzed the two clusters formed with drought: that affected only by drought and that affected by the presence of plants and drought. To facilitate the comparison between the results of analysis in each condition, we 
Table 1 Detailed information for the samples used to make Fig. 2 and Fig. 3

\begin{tabular}{|c|c|c|c|c|}
\hline Alias & Specific name & Treatment $^{a}$ & Plant $^{b}$ & Source project \\
\hline 1 & Watered treated soil from Arbuckle (U.S) & WATERED & $\mathrm{O}$ & PRJNA386367 \\
\hline 2 & Watered treated soil from Biggs (U.S) & WATERED & $\mathrm{O}$ & PRJNA386367 \\
\hline 3 & Watered treated soil from Davis (U.S) & WATERED & $\mathrm{O}$ & PRJNA386367 \\
\hline 4 & Drought treated soil from Arbuckle (U.S) & DROUGHT & $\mathrm{O}$ & PRJNA386367 \\
\hline 5 & Drought treated soil from Bigs (U.S) & DROUGHT & $\mathrm{O}$ & PRJNA386367 \\
\hline 6 & Drought treated soil from Davis (U.S) & DROUGHT & $\mathrm{O}$ & PRJNA386367 \\
\hline 7 & Watered treated irrigated soil (Thailand) & WATERED & $x$ & PRJNA362531 \\
\hline 8 & Watered treated rainfed soil (Thailand) & WATERED & $x$ & PRJNA362531 \\
\hline 9 & Drought treated irrigated soil (Thailand) & DROUGHT & $x$ & PRJNA362531 \\
\hline 10 & Drought treated rainfed soil (Thailand) & DROUGHT & $x$ & PRJNA362531 \\
\hline
\end{tabular}

All replicates in each sample are averaged. In case of alias 7 and alias 8, incubated condition data is used out of fresh, re-incubated, drought, and recovery aWATERED: well watered state; DROUGHT: water is drained from soil and dried state

${ }^{\mathrm{b}} \mathrm{O}$ : plant exists; $\mathrm{X}$ : plant does not exist

calculated the relative abundance ratio of drought to watered condition (from here "ratio").

Populations associated with drought conditions regardless of the presence of plants include Chloroflexi, Nitrospirae, Rokubacteria, and Bacteroidetes. In terms of the ratio of the abundance under drought to that under watered conditions, Chloroflexi shows a value of 0.738 . In case of Nitrospirae and Rokubacteria, the ratios are 0.389 and 0.455 , respectively (Table S8). Lastly, Bacteroidetes shows the lowest ratio of 0.250 (Table S8). Taken together, the microbiome developing under stress conditions is characterized by statistically significant shifts in the population that do not involve the establishment of a phylum with a relative abundance ratio greater than 1 . This indicates that population of all phyla described in this section decreased under drought conditions (Table S6, Table S8, Figure S2). Therefore, we did not further explore this cluster to identify useful candidate phyla to assist rice in overcoming drought stress.

A recent study reporting on Nitrospirae focused mainly on the nitrogen cycle (Xue et al. 2016); knowledge about its relationship with drought is limited. Similarly, drought-related information on Rokubacteria is limited, especially since this phylum has been newly classified (Becraft et al. 2017). Bacteroidetes make up the largest portion of the microbiome of mammalian

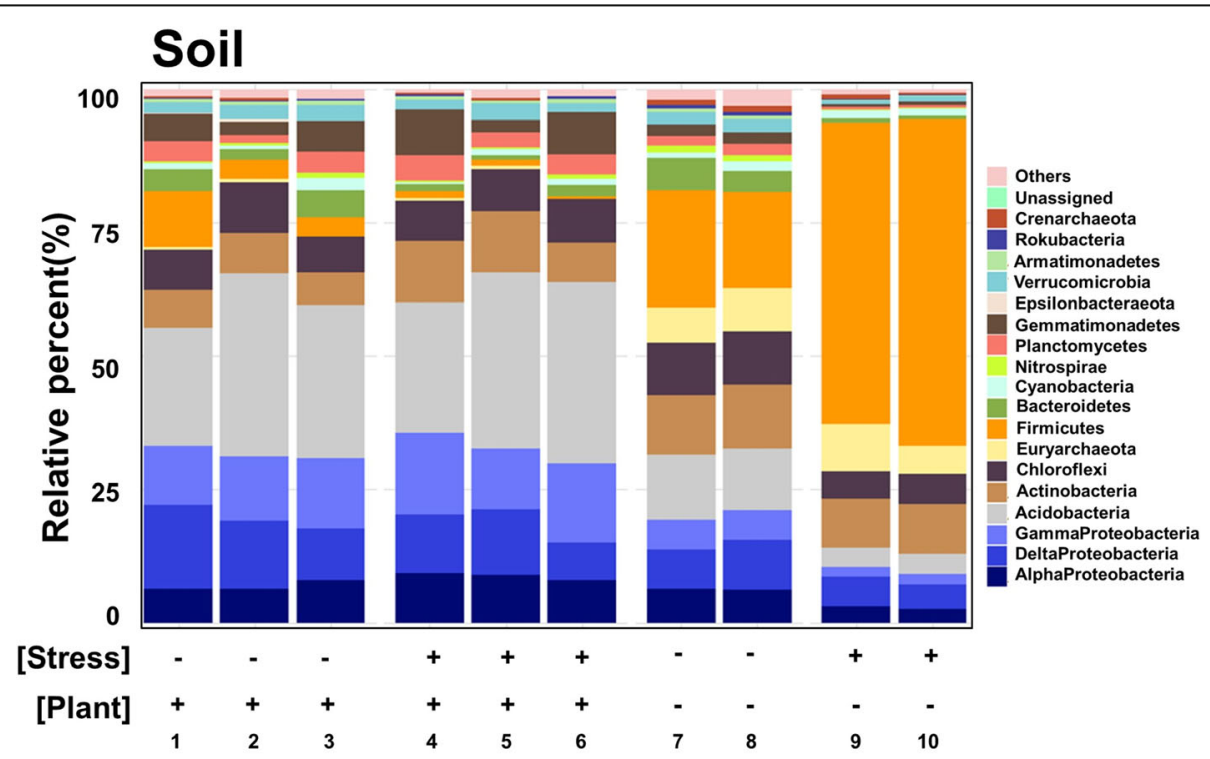

Fig. 2 Relative abundance of the 16 most abundant phyla from each soil sample collected from systems under drought stress or the presence of plants. The data points represent the abundance of each phylum relative to the total of all phyla in each sample. The 16 most abundant phyla in each treatment are integrated and identified, as well as an unassigned phylum. Only Proteobacteria is subdivided into classes. Clusters of other phyla are labeled as "Others." Grids are set according to the application of stress (drought (+)/watered (-)) and the presence of plants (with $(+) /$ without $(-))$. Detailed information on each sample is presented in Table 1 
intestines, thus its characteristics have been revealed through human microbiome studies. However, the interactions between plants and Bacteroidetes is largely unknown. Literature searches on all the phyla in this cluster provided no clues on their functional significance in relation to drought. Thus, our search for candidate phyla that can help plants overcome drought condition focused on the cluster consisting of populations that are affected by the presence of plants and stress.

The populations affected by both drought conditions and the presence of plants are Armatimonadetes, Verrucomicrobia, Firmicutes, and Proteobacteria. The Armatimonadetes ratios of drought to watered are 0.819 in the presence of plants and 0.219 in the absence of plant (Table S7). The reduction in the relative abundance of Armatimonadetes is consistent with previous results in potato (Gumiere et al. 2019). Similarly, the Verrucomicrobia ratios of drought to watered are 0.896 in the presence of plants and 0.378 in the absence of plants (Table S7). In the presence of plants, the abundance levels of populations of Armatimonadetes and Verrucomicrobia were lower under drought conditions than those under watered conditions. However, their population levels increased when they were interacting with plants under drought conditions, compared to their levels without this interaction. Therefore, these phyla have potential significance in assisting plants to overcome drought stress.

In the case of Firmicutes, the ratios of drought to watered are 0.161 in the presence of plants and 2.935 in the absence of plants (Table S7). This result that shows the ratio increasing under drought conditions in the absence of plants and is consistent with previous findings that members of the Firmicutes community increase under drought conditions (Chodak et al. 2015). In the case of Proteobacteria, the ratios of drought to watered are 1.029 in the presence of plants and 0.484 in the absence of plants. Considering that the $p$-value of ANOVAtest is less than 0.05 and the ratio is greater than 1 in the presence of plants, Proteobacteria is selected as the phylum that is most affected by drought conditions in the presence of plants (Table S6, Table S7). In addition, Proteobacteria are noticeably reduced under drought conditions in the absence of plants, an observation that is consistent with previous research showing that the abundance of Proteobacteria is very low ratio in water-limited desert soil (Bu et al. 2018) (Table S7). Although we did not find any reports indicating that Proteobacteria populations may shift in response to drought conditions in the presence of plants, we nevertheless selected Proteobacteria as the best candidate phylum to assist plants in overcoming drought stress. Thus, we performed more detailed analysis on this phylum.

To identify specific populations within Proteobacteria that respond to the presence of plants and drought, we analyzed the microbiome at the family level. The outcome of the analysis is presented in Fig. 3. The plot integrates 30 of the most abundant families in each sample. The following 13 families show a statistically meaningful

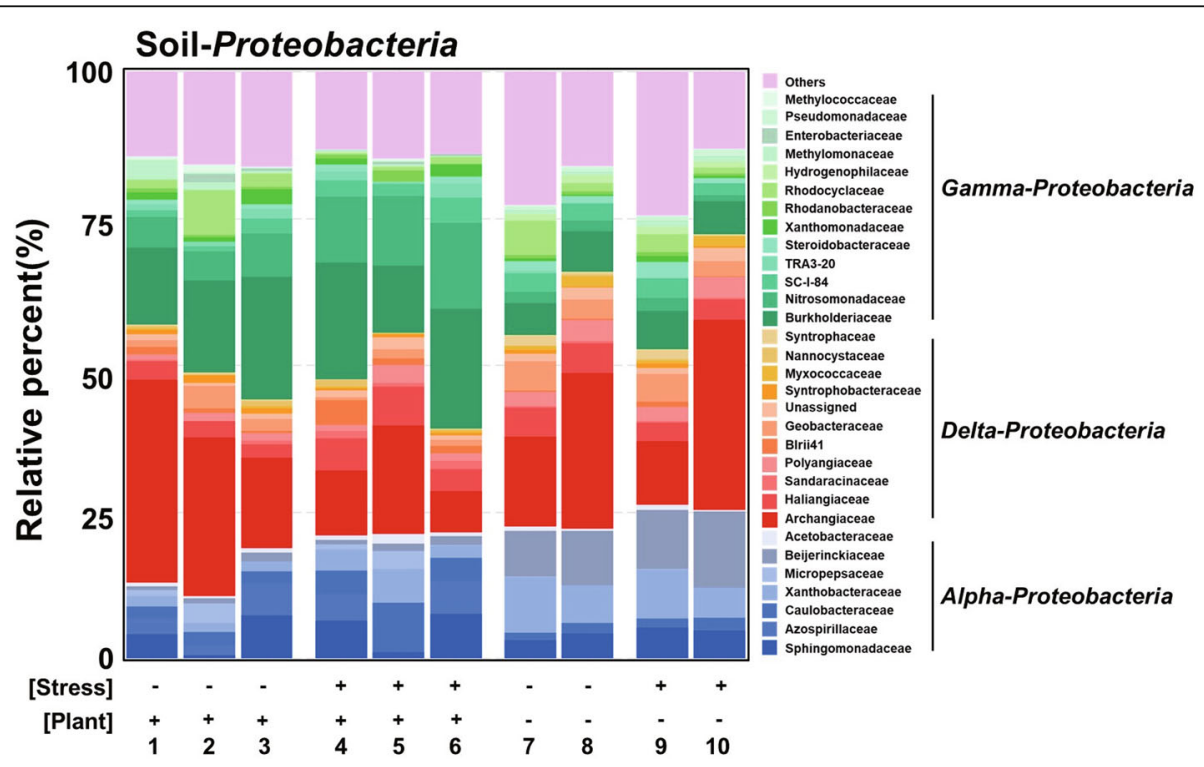

Fig. 3 Relative abundance of the 30 most abundant Proteobacteria families from soil samples differentiated according to presence of drought stress or plants. Data points represent the abundance of each family relative to the total abundance of all families in each sample. The 30 most abundant families in each treatment were integrated, resulting in the identification of 31 families (one group consists of unassigned families). Each color represents a microbe in the following classes: Alphaproteobacteria (blue); Deltaproteobacteria (red); and Gammaproteobacteria (green). Bacteria not belonging to these classes are clustered as "Others." Grids are set according to the application of stress (drought $(+) /$ watered $(-))$ and presence of plant (with (+)/without (-)). Detailed information of each sample is presented in Table 1 


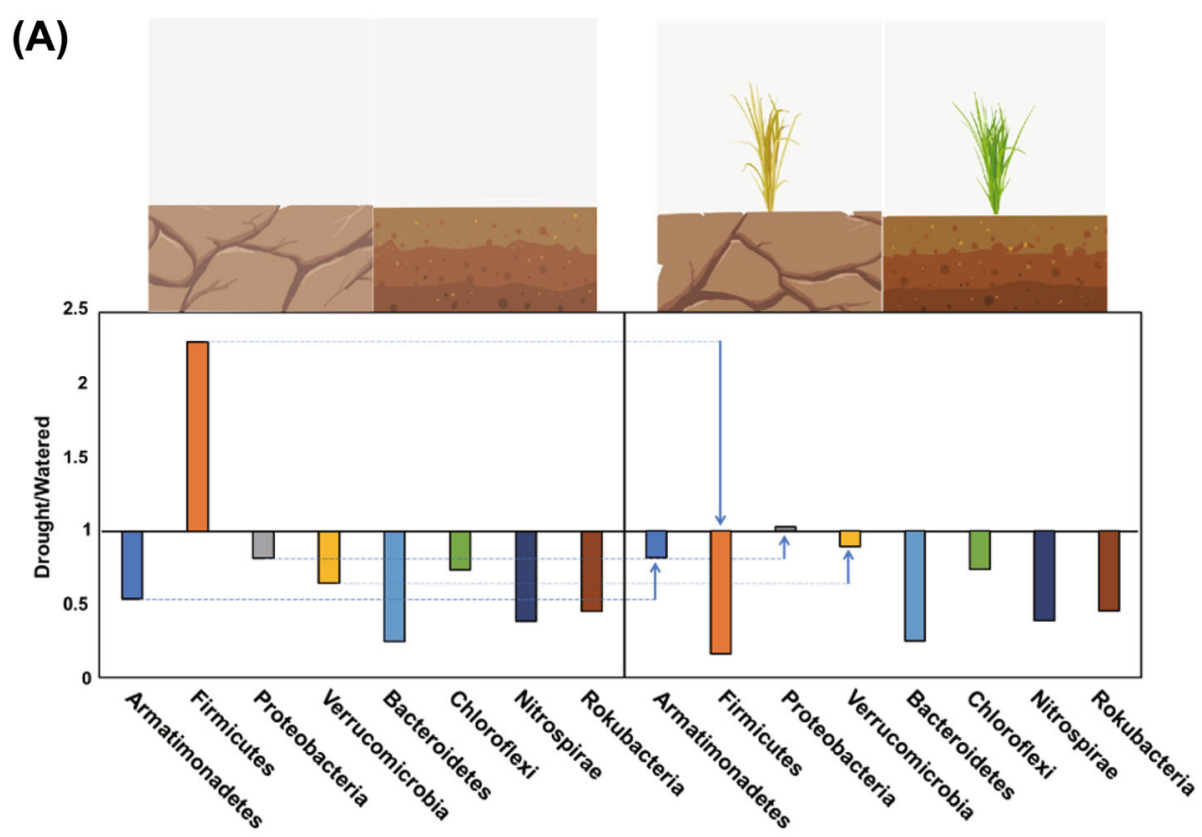

(B)

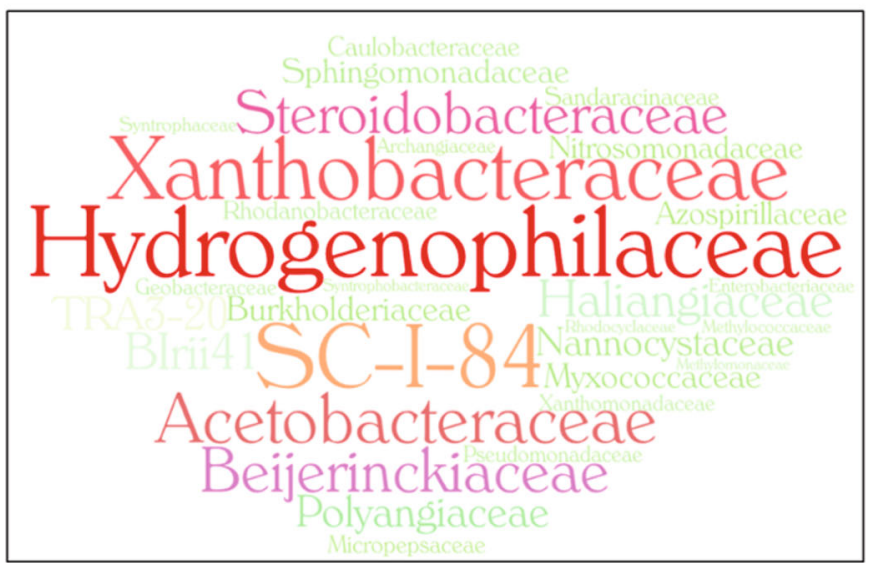

Fig. 4 Model of the microbiome shift following a change in soil state and the presence of plants. The microbial shift produces two distinct clusters and suggests the dominance of six families belong to Proteobacteria under drought condition. a The left panel shows the ratio of drought to watered condition in the absence of plant for eight phyla and right one shows the ratio in the presence of plant for eight phyla selected from two clusters affected by drought. When watered soil with plants shift to drought with plants, phyla showing significant population shift are indicated with vertical arrows and dotted lines ( $p$-value <0.05; two-way ANOVA of both factors) (Table S6). The x-axis represents the ratio of drought to watered condition and y-axis indicates eight phyla significantly affected by drought. $\mathbf{b}$ The WordCloud refers to 13 families within the Proteobacteria that shift significantly as a cluster in the presence of both drought stress and plants (Table S9). Six families have statistically significant $p$-values and are presented in bigger size of letters with different color; these exhibit the same pattern as Proteobacteria in response to drought conditions in the presence of plants. The word sizes indicate the relative abundance ratio under drought conditions

cluster shift $(P<0.05)$ in response to both drought and the presence of plants: Xanthobacteraceae, Acetobacteraceae, Beijerinckiaceae, Geobacteraceae, Syntrophobacteraceae, Syntrophaceae, SC-I-84, Xanthomonadaceae, Methylomonaceae, Rhodocyclaceae, Methylococcaceae, Steroidobacteraceae, and Hydrogenophilaceae (Table S9). Among these families, Xanthobacteraceae, Acetobacteraceae, Beijerinckiaceae, SC-I-84, Steroidobacteraceae, and Hydrogenophilaceae share similar trends as that of Proteobacteria (Table S10). Xanthobacteraceae is known to interact with plants and is involved in carbon and nitrogen cycling (Oren 2014; Wang et al. 2016). Meanwhile, members of Acetobacteraceae include plant growth promoting bacteria and well-known nitrogen-fixing bacteria (Saravanan et al. 2008; Reis and Teixeira 2015). In summary, we suggest six families belonging to Proteobacteria as candidates that assist plants cope with drought conditions.

Based on the results of our re-analysis, we constructed a model illustrating the statistically significant rice soil 
microbiome shift of four phyla in response to drought and the presence of plants; and four other phyla that are affected only by drought conditions. Figure 4 summarizes the abundance ratios of eight phyla affected by drought in the presence or absence of plants (Fig. 4a). Of these, Armatimonadetes, Verrucomicrobia, and Proteobacteria interacting with plants showed increased populations under drought conditions, while those of Firmicutes decreased. The Proteobacteria, in particular, includes more abundant populations under drought conditions than under watered conditions in the presence of plants, and was thus selected for the further analysis to identify six families that we propose as candidates that are worth further study (Fig. 4b). To help researchers in this field, we have analyzed the taxonomy of amplicons from reference studies that were excluded from our study due to insufficient amount of data (Figures S3, S4, and Table S11).

To compare the results of our analysis to those of other crops, we analyzed sorghum (Sorghum bicolor L.) data under drought (Figure S5). In this case, Proteobacteria did not display statistically significant population shifts. Thus, while additional experiments with Proteobacteria are recommended in rice, we cannot extend this recommendation to sorghum.

In conclusion, re-analysis of amplicon sequence data identifies several microbial shifts under drought conditions that depend on the presence of plants. We propose Proteobacteria as a suitable target for studies aiming to promote crop growth, in the same manner that previous studies have shown that inoculation of Actinobacteria enhances plant growth (Yandigeri et al. 2012; Hamedi and Mohammadipanah 2015). Because the composition of the microbiome depends largely on complex environmental variables and the genotype of the plant, new perspectives will be discovered as new microbiome data are generated.

\section{Supplementary information}

Supplementary information accompanies this paper at https://doi.org/10. 1186/s12284-020-00403-6.

Additional file 1:. Materials and Methods.

Additional file 2: Figure S1. Box plots of indexes for alpha diversity by drought condition (A) or by both factors (B) (Chao1, Faith_PD, Shannon, Simpson). Each index is calculated using the QIIME2 functions: qiime diversity alpha-phylogenetic and qiime diversity alpha.

Additional file 3: Figure S2. Bar plot of the integrated abundance of the 16 most dominant phyla in each environment. Each bar represents the mean value under each condition. Phyla that are not included in the integrated set of 16 phyla are classified as "others."

Additional file 4: Figure S3. Bar plot of the integrated abundance of the 16 most dominant phyla from endosphere data collected under drought stress. Each bar represents the mean value under each condition. The 16 most abundant phyla in each treatment are integrated, resulting in the identification of 16 phyla, including unassigned phyla. Only groups within the Proteobacteria phylum are presented as classes. Phyla other than those classified are clustered as "Others." Detailed information on each sample is presented in Table S11.
Additional file 5: Figure S4. Bar plot of the 16 most abundant phyla from rhizosphere and rhizoplane data collected under drought stress. Each bar represents the mean value under each condition. The 16 most abundant phyla in each treatment are integrated, resulting in the identification of 16 phyla, including unassigned phyla. Only groups within the Proteobacteria are presented as classes. Phyla other than those classified are clustered as "Others." Detailed information on each sample is presented in Table S11.

Additional file 6: Figure S5. Bar plot of the integrated abundance the 16 most abundant phyla from sorghum under drought conditions. Ctrl, XXX, and DR refer to Control, well-watered, and drought state, respectively. Samples were grown under well-watered condition for 4 weeks to establish roots and then water was drained at 5 weeks after planting. Drought-stressed plants were sampled every week starting from 5 weeks after planting. Control samples were collected from a well-watered bathtub.

Additional file 7: Table S1. All amplicon sequence data collected for re-analysis.

Additional file 8: Table S2. MinMax of samples used in the analysis. Additional file 9: Table S3. Median of the samples used in the analysis. Additional file 10: Table S4. Average of the samples used in the analysis.

Additional file 11: Table S5. Total count of samples used in the analysis.

Additional file 12: Table S6. $P$-value resulting from a two-way ANOVA accounting for drought state and presence of plant.

Additional file 13: Table S7. Relative abundance of the 16 most abundant phyla from each sample collected under drought in the presence of plants.

Additional file 14: Table S8. Relative abundance of the 16 most abundant phyla for each environmental condition.

Additional file 15: Table S9. $P$-value resulting in identification of families of Proteobacteria from a two-way ANOVA according to drought state and presence of plant.

Additional file 16: Table S10. Relative abundance of the 30 most abundant families of Proteobacteria from each sample according to drought state and plant presence.

Additional file 17: Table S11. Details regarding sample data used for Figures S3 and S4.

Abbreviations

ANOVA: Analysis of Variance; NGS: Next Generation Sequencing; OTU: Operational Taxonomic Unit

Acknowledgments

We appreciate Change21 Research program in Kyung Hee University.

\section{Authors' Contributions}

EJL, KHJ, WJH, and SWJ designed the work. MHY, WJH, and SWJ analyzed the microbiome data and generated figures and tables. All authors wrote and revised the manuscript. The authors read and approved the final manuscript.

\section{Funding}

This work was supported by grants from the Next Generation BioGreen 21 Program (PJ01325901 and PJ01366401 to KHJ); National Research Foundation of Korea (NRF) funded by the Korea government (MSIP) (2018R1A4A1025158 to KHJ, and 2016R1A2B2012424 to EJL), and Global Ph.D. Fellowship Program supported by the NRF (NRF-2018H1A2A1060336 to WJH).

\section{Availability of Data and Materials}

The datasets generated or analyzed in the current study are included in this article and its additional files. Detailed information about the all sequence data is mentioned in Table S1. All of OTU data we reanalyzed are prepared at https://github.com/WOOJONGHONG/Soil-Metagenome-reanalysis. 
Ethics Approval and Consent to Participate

Not applicable.

\section{Consent for Publication}

Not applicable.

\section{Competing Interests}

The authors declare they have no competing interests.

\section{Author details}

'Division of Life Sciences, College of Life Sciences and Biotechnology, Korea University, Seoul 02841, South Korea. ${ }^{2}$ Graduate School of Biotechnology, Kyung Hee University, Yongin 17104, South Korea. ${ }^{3}$ Graduate School of Biotechnology \& Crop Biotech Institute, Kyung Hee University, Yongin 17104, South Korea. ${ }^{4}$ Department of Oriental Medicine Biotechnology, College of Life Sciences, Kyung Hee University, Yongin 17104, South Korea.

Received: 28 July 2019 Accepted: 23 June 2020

Published online: 02 July 2020

\section{References}

Ahn J, Song J, Kim B, Kim M, Joa J, Weon H (2012) Characterization of the bacterial and archaeal communities in rice field soils subjected to long-term fertilization practices. J Microbiol 50:754-765

Arjun JK, Harikrishnan K (2011) Metagenomic analysis of bacterial diversity in the rice rhizosphere soil microbiome. Biotechnol Bioinf Bioeng 1:361-367

Becraft ED, Woyke T, Jarett J, Ivanova N, Godoy-Vitorino F, Poulton N, Brown JM, Brown J, Lau M, Onstott T (2017) Rokubacteria: genomic giants among the uncultured bacterial phyla. Front Microbiol 8:2264

Berg G, Grube M, Schloter M, Smalla K (2014) Unraveling the plant microbiome: looking back and future perspectives. Front Microbiol 5:148

Brencic A, Winans SC (2005) Detection of and response to signals involved in host-microbe interactions by plant-associated bacteria. Microbiol Mol Biol Rev 69:155-194

Bu X, Gu X, Zhou X et al (2018) Extreme drought slightly decreased soil labile organic $\mathrm{C}$ and $\mathrm{N}$ contents and altered microbial community structure in a subtropical evergreen forest. For Ecol Manag 429:18-27

Chodak M, Gołębiewski M, Morawska-Płoskonka J et al (2015) Soil chemical properties affect the reaction of forest soil bacteria to drought and rewetting stress. Ann Microbiol 65:1627-1637

Edwards J, Johnson C, Santos-Medellin C, Lurie E, Podishetty NK, Bhatnagar S, Eisen JA, Sundaresan V (2015) Structure, variation, and assembly of the rootassociated microbiomes of rice. Proc Natl Acad Sci U S A 112:E911-E920

Germida JJ, Siciliano SD, Renato de Freitas J, Seib AM (1998) Diversity of rootassociated bacteria associated with field-grown canola (Brassica napus L.) and wheat (Triticum aestivum L.). FEMS Microbiol Ecol 26:43-50

Gumiere T, Gumiere SJ, Matteau J-P. Constant P, Létourneau G, Rousseau AN (2019) Soil bacterial community associated with high potato production and minimal water use. Front Environ Sci 6:161

Hamedi J, Mohammadipanah F (2015) Biotechnological application and taxonomical distribution of plant growth promoting actinobacteria. J Ind Microbiol Biotechnol 42(2):157-171

Ikeda S, Sasaki K, Okubo T, Yamashita A, Terasawa K, Bao Z, Liu D, Watanabe T, Murase J, Asakawa S (2014) Low nitrogen fertilization adapts rice root microbiome to low nutrient environment by changing biogeochemical functions. Microbes Environ 29:50-59

Lethbridge G, Davidson M (1983) Root-associated nitrogen-fixing bacteria and their role in the nitrogen nutrition of wheat estimated by $15 \mathrm{~N}$ isotope dilution. Soil Biol Biochem 15:365-374

Lugtenberg B, Kamilova F (2009) Plant-growth-promoting rhizobacteria. Annu Rev Microbiol 63:541-556

Oren A (2014) The family Xanthobacteraceae. In: Rosenberg E, DeLong EF, Lory S et al (eds) The prokaryotes: Alphaproteobacteria and Betaproteobacteria. Springer, Berlin, pp 709-726

Peng S, Huang J, Sheehy JE, Laza RC, Visperas RM, Zhong X, Centeno GS, Khush GS, Cassman KG (2004) Rice yields decline with higher night temperature from global warming. Proc Natl Acad Sci U S A 101:9971-9975

Reim A, Hernández M, Klose M, Chidthaisong A, Yuttitham M, Conrad R (2017) Response of methanogenic microbial communities to desiccation stress in flooded and rain-fed paddy soil from Thailand. Front Microbiol 8:785
Reis VM, Teixeira KR (2015) Nitrogen fixing bacteria in the family Acetobacteraceae and their role in agriculture. J Basic Microbiol 55:931-949

Rothenberg SE, Anders M, Ajami NJ, Petrosino JF, Balogh E (2016) Water management impacts rice methylmercury and the soil microbiome. Sci Total Environ 572:608-617

Santos-Medellín C, Edwards J, Liechty Z, Nguyen B, Sundaresan V (2017) Drought stress results in a compartment-specific restructuring of the rice rootassociated microbiomes. MBio 8:e00764-e00717

Saravanan VS, Madhaiyan M, Osborne J et al (2008) Ecological occurrence of Gluconacetobacter diazotrophicus and nitrogen-fixing Acetobacteraceae members: their possible role in plant growth promotion. Microb Ecol 55:130-140

Sessitsch A, Hardoim P, Döring J, Weilharter A, Krause A, Woyke T, Mitter B, Hauberg-Lotte L, Friedrich F, Rahalkar M (2012) Functional characteristics of an endophyte community colonizing rice roots as revealed by metagenomic analysis. Mol Plant-Microbe Interact 25:28-36

Wang W, Wang H, Feng Y et al (2016) Consistent responses of the microbial community structure to organic farming along the middle and lower reaches of the Yangtze River. Sci Rep 6:35046

Xue C, Penton CR, Zhang B, Zhao M, Rothstein DE, Mladenoff DJ, Forrester JA, Shen Q, Tiedje JM (2016) Soil fungal and bacterial responses to conversion of open land to short-rotation woody biomass crops. GCB Bioenergy 8:723-736

Yandigeri MS, Meena KK, Singh D et al (2012) Drought-tolerant endophytic actinobacteria promote growth of wheat (Triticum aestivum) under water stress conditions. Plant Growth Regul 68(3):411-420

\section{Publisher's Note}

Springer Nature remains neutral with regard to jurisdictional claims in published maps and institutional affiliations.

\section{Submit your manuscript to a SpringerOpen ${ }^{\circ}$ journal and benefit from:}

- Convenient online submission

- Rigorous peer review

- Open access: articles freely available online

- High visibility within the field

- Retaining the copyright to your article

Submit your next manuscript at $\boldsymbol{\nabla}$ springeropen.com 